\title{
Anorexia nervosa through the looking glass of the draft ICD-11 diagnostic criteria: a disorder in transition
}

\author{
Nadia Micali $^{1,2} \cdot$ Johannes Hebebrand $^{3}$
}

Published online: 26 September 2015

(C) Springer-Verlag Berlin Heidelberg 2015

This issue of European Child and Adolescent Psychiatry (ECAP) includes the first ESCAP (European Society of Child and Adolescent Psychiatry) expert article authored by Herpertz-Dahlmann and colleagues [12] with a focus on clinical practice related to anorexia nervosa in Europe. Authors of ESCAP expert articles are invited by the ESCAP board to assemble a group of authors representing European diversity to compile an article illustrating current understanding, diagnostic issues and treatment of a particular mental disorder of childhood and adolescence. These peer-reviewed articles will appear as they are accepted in ECAP; they serve to provide the readers with an excellent overview of the respective disorder/topic written by distinguished researchers. The articles additionally serve to illustrate the mutually beneficial collaboration between the society and this journal.

In their expert article Herpertz-Dahlmann et al. initially discuss the novel DSM-5 diagnostic criteria [2] for anorexia nervosa and briefly highlight the corresponding ICD-11 beta draft (WHO, ICD-11 beta draft [21]), thus providing an opportunity to further elaborate on these proposed criteria.

The diagnostic criteria of a mental disorder are frequently taken for granted; they are usually valid for prolonged periods of time and are virtually known by heart by

Johannes Hebebrand

johannes.hebebrand@uni-due.de

1 Department of Psychiatry, Icahn School of Medicine at Mount Sinai, New York, NY, USA

2 Institute of Child Health, University College London, London, UK

3 Department of Child and Adolescent Psychiatry, Psychosomatics, and Psychotherapy, University Hospital Essen, University of Duisburg-Essen, Essen, Germany professionals dealing with the respective patients clinically and/or research-wise. Moreover, diagnostic criteria for mental (psychiatric) disorders have traditionally resulted from clinical observations, rather than empirical studies. A degree of conservatism is warranted to maintain the core features of a disorder over time; however, the emergence of relevant novel findings with an impact on the diagnosis of a disorder merits consideration, thus entailing evolution of the diagnostic criteria over time. As such, it is important to realize that despite our more or less fixed clinical perception of a particular disorder at any time point, the diagnosis is nevertheless fluid if viewed over a period of several decades.

Anorexia nervosa is a disorder in transition. DSM-IV [1] still described this eating disorder as stemming from the 'refusal' of a patient to maintain her/his body weight within the normal range. This concept originated over 140 years ago, when in 1873 Lasègue [15] described that at the end of some weeks of initial illness characterized by feelings of "uneasiness after food, vague sensations of fullness, suffering and gastralgia post prandium", "refusal of food" sets in "that may be indefinitely prolonged". The reasons underlying this conceptualization are seemingly rooted in our belief that eating is a basic need, which requires a wilful, deliberate action to be overridden to such an extent as to compromise health and reproduction by induction of underweight and starvation. In addition, we speculate that both our fascination and frustration with these patients leaves us with no other explanation than that 'refusal' represents the core feature of the disorder, and along similar lines that the patients are in "denial of the seriousness of the current low body weight" (DSM-IV). Unfortunately, this speculation has highly influenced lay views of anorexia nervosa, resulting in public perception that individuals with anorexia nervosa are to blame for wilfully 'causing' their 
Table 1 ICD-11 beta draft (WHO) [21]

Anorexia nervosa is characterized by significantly low body weight for the individual's height, age and developmental stage [body mass index (BMI) less than $18.5 \mathrm{~kg} / \mathrm{m} 2$ in adults and BMI for age under fifth percentile in children and adolescents] that is not due to another health condition or to the unavailability of food. Low body weight is accompanied by a persistent pattern of behaviours to prevent restoration of normal weight, which may include behaviours aimed at reducing energy intake (restricted eating), purging behaviours (e.g. self-induced vomiting, misuse of laxatives), and behaviours aimed at increasing energy expenditure (e.g. excessive exercise), typically associated with a fear of weight gain. Low body weight or shape is central to the person's self-evaluation or is inaccurately perceived to be normal or even excessive.

disorder. Moreover, this conceptualization was at odds with the clinical presentation of anorexia nervosa in children and adolescents, who might be unable to articulate 'refusal' and would therefore not have fulfilled criteria for a diagnosis of anorexia nervosa [16]. In 2004, Hebebrand and coworkers initially [9] and since then repeatedly [8, 5] criticized this conception by pointing out that empirical evidence for the terms refusal and denial is lacking. In addition, these terms convey a paternalistic and pejorative attitude towards affected individuals. Both terms were subsequently dropped in DSM-5 [2].

It is of interest to point out that the ICD 10 diagnostic criteria for research [19] had defined anorexia nervosa in a less stigmatizing way, but also clearly portrayed the idea that the disorder, and in particular weight loss, is "selfinduced". A comparison with diagnostic criteria for most other mental disorders readily reveals that such a 'selfinduction' is not a common theme and presumably reflects our inability to view a reduced energy intake and ensuing weight loss in these patients as not being intentional.

The ICD-11 beta draft version (see Table 1) [21] is to be commended, because the criteria are based on clearly observable behaviours and cognitions [8, 9]. It tries to avoid statements as to the intentions of a patient. Thus, ICD-11 merely refers to underweight circumscribed as a "significantly low body weight for the individual's height, age and developmental stage" (phrasing adapted from DSM-5), which cannot be explained by another "health condition or to the unavailability of food". In contrast, DSM-5 perceives "restriction of energy intake relative to requirements" as a core aspect of the disorder. In our opinion, it is indeed important to make reference to the motor hyperactivity (e.g. excessive exercise) observed in a subgroup of patients. However, the ICD-11 beta draft version infers causality by stating that the "low body weight is accompanied by a persistent pattern of behaviours to prevent restoration of normal weight, which may include behaviours aimed at reducing energy intake (restricted eating), purging behaviours (e.g. self-induced vomiting, misuse of laxatives), and behaviours aimed at increasing energy expenditure (e.g. excessive exercise)". As strange as it may seem, the empirical evidence that all patients with anorexia nervosa-at all ages and at all stages of the disorder-show these behaviours to prevent restoration of normal weight is lacking [9]. On the contrary, hypoleptinemia clearly drives hyperactivity in a rodent model of anorexia nervosa [4]; in patients, lower leptin levels have also been associated with higher activity levels [10, 13]. Both findings indicate that hyperactivity in patients with anorexia nervosa is not necessarily a result of the patients' intention to prevent restoration of normal weight, but may very well represent an endocrinologically induced behaviour. Indeed, we have speculated that excessive hyperactivity (and potentially other behaviours and cognitions) in these patients may be amenable to treatment with recombinant leptin [7].

ICD-11, again in contrast to DSM-5, explicitly provides a definition of "a significantly low body weight" for adults based on the WHO classification of underweight $\left(\mathrm{BMI}<18.5 \mathrm{~kg} / \mathrm{m}^{2}\right.$; WHO [20]) and for children and adolescents based on a BMI below the 5th age percentile. The provision of thresholds will prove important in increasing the diagnostic reliability; in contrast, DSM-5 intentionally avoided a clear-cut weight threshold. As for all dimensional somatic or psychological traits, clear-cut thresholds are helpful for clinicians and patients. Obviously, weight-forheight below the specified consensus is but one criterion for the diagnosis of anorexia nervosa. As such, a too restrictive weight criterion might lead clinicians to resort to a subthreshold category for many seriously ill patients, such as was the case with Eating Disorder Not Otherwise Specified in DSM-IV. A BMI of $18.5 \mathrm{~kg} / \mathrm{m}^{2}$ at age 18 , the beginning of adulthood, roughly corresponds to the 10th BMI age percentile [8]. As such, the ICD-11 weight criterion proposed for patients below age 18 is potentially too strict. Furthermore, to keep in line with the ICD-10 weight criterion ("body weight of at least $15 \%$ below the normal"), which corresponds to BMI values in the range between the 5th and 10th age percentiles [11], the less strict cutoff would appear more appropriate. A substantial percentage of patients currently diagnosed with anorexia nervosa presents with a BMI between the 5th and 10th percentiles. Importantly, if a child or young adolescent presents with a BMI between the 5th and 10th age percentiles, one might want to appropriately diagnose anorexia nervosa, enabling prompt recognition and early intervention. A too restrictive weight threshold would enable diagnosis of anorexia nervosa (and possibly treatment) only once a child/adolescent 
is severely underweight, therefore potentially hampering recovery. Finally, in therapeutic terms, it is likely disadvantageous to define a too low threshold for the weight criterion: patients as well as their parents could wrongly define recovery via this threshold. In conclusion, a higher threshold for the weight criterion (i.e., to the 10th percentile) for children and adolescents is a must!

Importantly, ICD-10 without stating the term starvation acknowledges a "widespread endocrine disorder", which includes but goes well beyond amenorrhoea (see criterion 4). Interestingly, DSM-5 no longer refers to endocrine alterations; the former $\mathrm{D}$ criterion, the requirement of amenorrhoea in postmenarcheal females, was dropped. The only indirect reference to the "widespread endocrine disorder", which can be life threatening in medical terms, is made in the DSM-5 A criterion: "Restriction of energy intake relative to requirements leading to a significantly low body weight in the context of age, sex, developmental trajectory, and physical health". ICD-11 now also avoids any reference to the symptoms of starvation and the associated endocrine disturbances. We view this as inappropriate, because symptoms of starvation separate females with AN from constitutionally underweight females, who account for most of those females with a BMI below $18.5 \mathrm{~kg} / \mathrm{m}^{2}$ or a BMI < the fifth or tenth BMI age percentile. Does it no longer hold true that the core symptomatology of anorexia nervosa consists of an intertwining of primary behavioural features and cognitions with mental and physical symptoms related to starvation, which differ depending on age and stage of the disorder $[8,5]$ ? As such, we would welcome a reference in ICD-11 to the fact that anorexia nervosa features symptoms of starvation. Obviously, in light of attempts to define biological markers for mental disorders, the non-recognition of endocrine alterations associated with anorexia nervosa including for instance hypoleptinemia $[5,6]$ represents a step backwards.

Lastly, DSM-5 moved away from fear of weight gain/ fat phobia being a necessary criterion for the diagnosis of anorexia nervosa, by including engagement in persistent behaviour that interferes with weight gain as an alternative to fear of gaining weight or fear of fatness. This change mostly stemmed from evidence that a substantial proportion of patients with anorexia nervosa do not endorse fear of gaining weight/fatness. In particular, there is evidence that non-Western and younger presentations are less likely to express fear of gaining weight/fatness $[18,3]$. This shift is therefore particularly important for children and young adolescents. Comparable importance to behavioural indicators is also reflected in the beta draft ICD-11 criteria. The importance of weight and shape on self-evaluation was retained in DSM-5 and ICD-11, although similarly to fear of weight gain/fatness its validity in younger populations has been questioned. Multi-informant assessment can be very valuable in younger ages $[17,14]$, but its valuable role is not highlighted in the revisions.

In summary, both diagnostic manuals in their recent (DSM-5) and upcoming (ICD-11) revisions have taken into account theoretical approaches and evidence from clinical and epidemiological findings to refine and adapt diagnostic classifications to an increased understanding of the pathophysiology and variety of clinical presentations of anorexia nervosa. The orientation towards observable behaviour and cognitions represents a major step forward and is in accordance with the currently descriptive classifications of mental disorders. Whilst findings from neuroscience, genetics, epidemiology and cross-cultural nosology can help us deepen our understanding of the underlying pathophysiology of AN, how we define and 'measure' disorders will impact in a circular fashion on this understanding. In this respect, it is crucial to clarify if underweight associated with symptoms of starvation represents a cardinal feature of the disorder. Symptoms of starvation can also become manifest in individuals with overweight or even obesity, who have for instance fasted for a prolonged period of time and thus lost a substantial amount of body weight. Further research into anorexia nervosa like clinical presentations of normal weight and overweight subjects is warranted. Because both DSM-5 and ICD-11 agree that underweight is a physical symptom of the disorder, we plead for a minimally strict weight threshold that should be defined unambiguously (weight phobia should obviously NOT spread to the diagnosticians). Similarly, if we continue to infer intentions of patients, we need to have compiled the relevant evidence. It is important to realize that non-empirically substantiated views should not continue to shape our concept of this disorder. Ascertaining whether presentations of anorexia nervosa, particularly at younger ages (when the disorder first manifests), are well captured by current definitions is likely to not only result in prompt treatment provision and improving outcomes, but also in targeting treatment approaches to specific presentations. Similarly, experimental findings might shed light on whether anorexia nervosa as we know it really is a circumscribed specific phenotype rather than a common physiological expression of several underlying phenotypes.

\section{References}

1. American Psychiatric Association (1994) Diagnostic and Statistical Manual of Mental Disorders, 4th edn. Washington, DC

2. American Psychiatric Association (2013) Diagnostic and Statistical Manual of Mental Disorders, 5th edn. Washington, DC

3. Bravender T, Bryant-Waugh R, Herzog D, Katzman D, Kriepe RD, Lask B, Le Grange D, Lock J, Loeb KL, Marcus MD, Madden S, Nicholls D, O’Toole J, Pinhas L, Rome E, Sokol-Burger M, Wallin U, Zucker N (2010) Workgroup for classification of 
eating disorders in children and adolescents. classification of eating disturbance in children and adolescents: proposed changes for the DSM-V. Eur Eat Disord Rev 18(2):79-89

4. Exner C, Hebebrand J, Remschmidt H, Wewetzer C, Ziegler A, Herpertz S, Schweiger U, Blum WF, Preibisch G, Heldmaier G, Klingenspor M (2000) Leptin suppresses semi-starvation induced hyperactivity in rats: implications for anorexia nervosa. Mol Psychiatry 5(5):476-481

5. Föcker M, Knoll S, Hebebrand J (2013) Anorexia nervosa. Eur Child Adolesc Psychiatry 22(Suppl 1):S29-S35

6. Föcker M, Timmesfeld N, Scherag S, Bühren K, Langkamp M, Dempfle A, Sheridan EM, de Zwaan M, Fleischhaker C, Herzog W, Egberts K, Zipfel S, Herpertz-Dahlmann B, Hebebrand J (2011) Screening for anorexia nervosa via measurement of serum leptin levels. J Neural Transm 118(4):571-578

7. Hebebrand J, Albayrak Ö (2012) Leptin treatment of patients with anorexia nervosa? The urgent need for initiation of clinical studies. Eur Child Adolesc Psychiatry 21(2):63-66

8. Hebebrand J, Bulik CM (2011) Critical appraisal of the provisional DSM-5 criteria for anorexia nervosa and an alternative proposal. Int J Eat Disord 44(8):665-678

9. Hebebrand J, Casper R, Treasure J, Schweiger U (2004) The need to revise the diagnostic criteria for anorexia nervosa. J Neural Transm 111(7):827-840

10. Hebebrand J, Exner C, Hebebrand K, Holtkamp C, Casper RC, Remschmidt H, Herpertz-Dahlmann B, Klingenspor M (2003) Hyperactivity in patients with anorexia nervosa and in semistarved rats: evidence for a pivotal role of hypoleptinemia. Physiol Behav 79(1):25-37

11. Hebebrand J, Himmelmann GW, Heseker H, Schafer H, Remschmidt H (1996) Use of percentiles for the body mass index in anorexia nervosa: diagnostic, epidemiological, and therapeutic considerations. Int J Eat Disord 19(4):359-369

12. Herpertz-Dahlmann et al. (2015) ESCAP Expert Paper: New developments in the diagnosis and treatment of adolescent anorexia nervosa - a European perspective. Eur Child Adolesc Psychiatry. doi:10.1007/s00787-015-0748-7

13. Holtkamp K, Herpertz-Dahlmann B, Hebebrand K, Mika C, Kratzsch J, Hebebrand J (2006) Physical activity and restlessness correlate with leptin levels in patients with adolescent anorexia nervosa. Biol Psychiatry 60(3):311-313

14. House J, Eisler I, Simic M, Micali N (2008) Diagnosing eating disorders in adolescents: a comparison of the eating disorder examination and the development and well-being assessment. Int J Eat Disord 41(6):535-541

15. Lasegue EC (1873/1997) On hysterical anorexia. Obesity Res 5: 492-497

16. Nicholls D, Chater R, Lask B (2000) Children into DSM don't go: a comparison of classification systems for eating disorders in childhood and early adolescence. Int J Eat Disord 28(3):317-324

17. Swanson SA, Horton NH, Sonneville K, Crosby R, Eddy K, Field AE, Micali N (2014) Assessing eating disorder symptoms in adolescents utilizing multiple informants: results from the avon longitudinal study of parents and their children. Int $\mathrm{J}$ Eat Disord 47(5):475-482

18. Thomas JJ, Eddy KT, Ruscio J, Ng KL, Casale KE, Becker AE, Lee S (2015) Do recognizable lifetime eating disorder phenotypes naturally occur in a culturally Asian population? A combined latent profile and taxometric approach. Eur Eat Disord Rev. 23(3):199-209

19. WHO (1993) http://www.who.int/classifications/icd/en/GRNBOOK.pdf. Accessed 15 Sept 2015

20. WHO Global Database on Body Mass Index. http://apps.who.int/ bmi/index.jsp?introPage=intro_3.html. Accessed 15 Sept 2015

21. WHO ICD-11 Beta Draft. http://apps.who.int/classifications/ icd11/browse/l-m/en\#/http\%3a\%2f\%2fid.who.int\%2ficd\%2fentit y\%2f263852475. Accessed 15 Sept 2015 